\title{
Análisis de la composición proximal y potencial insecticida de la semilla de guanábana (Annona muricata L.) para el control del gusano cogollero del maiz (Spodoptera frugiperda J. E. Smith)
}

\author{
Lic. María Guerra Blandino \\ Tesista \\ Laboratorio de Biotecnología, UNAN-Managua \\ rucsg@hotmail.com
}

\section{Lic. José Poveda Suárez}

Tesista

Laboratorio de Biotecnología, UNAN-Managua jramongb54132@gmail.com

\section{M.Sc. Samantha Miranda Calero \\ Investigadora \\ Laboratorio de Biotecnología, UNAN-Managua smiranda@unan.edu.ni}

\section{M.Sc. Ena Rivers Carcache}

Investigadora

Laboratorio de Biotecnología, UNAN-Managua erivers@unan.edu.ni

\author{
M.Sc. Juan Ruíz Urbina \\ Investigador \\ Laboratorio de Biotecnología, UNAN-Managua \\ jcruiz77@yahoo.es
}

\section{Dra. Martha Lacayo Romero \\ Investigadora \\ Laboratorio de Biotecnología, UNAN-Managua biotecnologia@unan.edu.ni}

\section{Dra. Fátima Bolaños Ortega \\ Investigadora Instituto Nicaragüense de Tecnología Agropecuaria (INTA) agroalimentos@inta.gob.ni}

Ing. Henry Pedroza Chamorro

Investigador Instituto Nicaragüense de Tecnología Agropecuaria (INTA) hpedroza27@gmail.com

Fecha de recepción: 13 de febrero, 2020 / Fecha de aceptación: 19 de febrero, 2020 https://doi.org/10.5377/torreon.v9i24.9722

Palabras clave: Annona muricata L., composición proximal, guanábana, potencial insecticida, Spodoptera frugiperda 


\section{RESUMEN}

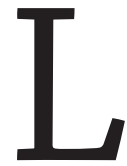

a guanábana (Annona muricata L.) es una especie tropical de interés mundial creciente debido a sus múltiples propiedades y sus aplicaciones en el campo de la salud y la agricultura. A fin de contribuir con el conocimiento del acervo existente y promover su utilización en la solución de problemáticas nacionales la presente investigación analizó la composición proximal y el potencial insecticida de las semillas de guanábana sobre el gusano cogollero (Spodoptera frugiperda J. E. Smith), un lepidóptero que amenaza el cultivo de maíz, uno de los rubros de mayor importancia en el país. En la investigación se incluyeron frutos disponibles en el comercio local clasificándolos según marcadores morfológicos internacionales. La composición proximal de las muestras de harina de semilla se determinó utilizando las metodologías descritas por Association of Official Analytical Chemists (AOAC) obteniendo como resultado: $4.29 \%$ de humedad, $95.71 \%$ de materia seca, $1.34 \%$ de cenizas, $31.14 \%$ de extracto etéreo, $2.59 \%$ de nitrógeno y $16.19 \%$ de proteína. Para la determinación del potencial insecticida se realizó un bioensayo (48 horas) donde se evaluó el efecto de los extractos de semilla completa y endospermo obtenidos mediante el método Soxhlet sobre el tercer estadio larval de gusanos cogolleros criados en el laboratorio desde su eclosión. Los extractos fueron aplicados al alimento (hojas de maíz) y se establecieron cinco concentraciones entre 0.70-25.00\%. En endospermo la concentración letal media (CL50) fue de $2.13 \%$, mientras que para semilla completa fue $1.85 \%$. En ambos extractos la mayor mortalidad se reportó a las 8 horas posterior a la aplicación.

\section{INTRODUCCIÓN}

Nicaragua es un país eminentemente agrícola con aproximadamente 1,504,000 ha de suelos arables (FAO, 2019) los cuales se encuentran destinados para el cultivo de diferentes rubros, entre ellos los cereales que constituyen un eslabón fundamental para la población. El maíz es probablemente el cereal de más importancia en el país ya que forma parte esencial de nuestra dieta y es utilizada para la elaboración de concentrados o piensos para animales (Bornemann et al., 2012).

La producción nacional del maíz se ve afectada por diversas plagas, siendo la principal el gusano cogollero (Spodoptera frugiperda J. E. Smith), un insecto lepidóptero que en algunos casos puede causar la pérdida total de la plantación cuando ocurre como gusano ejército (Jaramillo D., Á., Jaramillo G., O., Bustillo P., A., \& Gómez L., H., 1989). De igual manera, esta plaga es considerada persistente debido a que una vez que la fuente de alimento se agota, el gusano se traslada a nuevas fuentes donde se alimenta hasta completar su desarrollo.

Usualmente esta plaga es controlada con productos sintéticos como el nematicida Counter 10 G (terbufos), el insecticida órganofosforado Lorsban 4 E.C y piretroides como la Cipermetrina combinada con Diazinon (Espinoza et al., 1999; INTA, 2010), que perjudican la 
salud de los productores y sus familias, además de contaminar el ambiente y los alimentos en los que se aplican. En Nicaragua la incidencia de intoxicaciones asociadas a plaguicidas constituye una prioridad de salud pública dado la alta incidencia y mortalidad que representa (Pardo Abdala, L.M., Pérez Rodríguez, S., \& Gámez Bacallao, A., 2017). Adicionalmente, un daño colateral de estos productos es la afectación a especies u organismos no perjudiciales o beneficiosos para el ecosistema nativo del sitio de cultivo.

En este contexto y con el objetivo de reducir el impacto negativo del tratamiento químico tradicional, existe una tendencia internacional en buscar alternativas más ecológicas y sustentables que incluye la utilización de extractos de plantas para el control plagas. Dentro de este grupo de plantas con potencial insecticida encontramos a la guanábana (Annona muricata L.) cuyas propiedades se asocian a las acetogeninas, metabolitos secundarios responsables de sus características y presentes en todo el árbol (Arroyo et al., 2005; Bobadilla et al., 2005).

En Nicaragua la guanábana es conocida popularmente por el sabor de su pulpa y su consumo como fruta fresca. Las hojas y las semillas son usadas en medicina tradicional por su capacidad antitumoral, parasiticida y antidiarreica (Santos Pimenta et al., 2003). A nivel internacional su importancia económica ha crecido para proyectarse en la industria de la perfumería, farmacología y agrícola.

Aunque existen estudios a nivel internacional, para ampliar las aplicaciones de la guanábana nacional como materia prima se hace imperativo su caracterización morfológica y la determinación de su composición proximal debido a que las condiciones edafoclimáticas influyen directamente en la cantidad y calidad de los cultivares así como en su contenido nutricional (Peckham G., 1979). La presente investigación pretende contribuir con este fin al establecer pruebas de toxicidad (bioensayos) que evalúen el potencial insecticida de la guanábana sobre el gusano cogollero y determinando la composición proximal de las semillas para promover su aprovechamiento como materia prima.

\section{MATERIALES Y MÉTODOS}

\section{Colecta de las muestras}

Se colectaron 30 frutos en el comercio de los departamentos de Masaya y Carazo durante los meses de julio y agosto tomando en cuenta su estado de madurez fisiológica. Las muestras fueron transportadas al Laboratorio de Biotecnología de la Universidad Nacional Autónoma de Nicaragua, Managua (UNAN-Managua) donde se desinfectaron y procesaron en condiciones controladas para evitar su degradación. Las semillas fueron extraídas del fruto, se lavaron con agua potable y agua destilada, se secaron a temperatura ambiente durante 7 días y en horno de convección a $40^{\circ} \mathrm{C}$ durante 24 horas. 
Una porción de las semillas secas fueron separadas del pericarpio a fin de liberar el endospermo para analizar la concentración de los componentes insecticidas tanto del endospermo como en la semilla completa. Finalmente ambas muestras fueron maceradas y homogenizadas en un molino Retsch con tamiz integrado (tamaño de poro de $0.5 \mathrm{~mm}$ ). La harina obtenida se almacenó en recipientes herméticos color ámbar a $4^{\circ} \mathrm{C}$.

\section{Caracterización morfológica de las muestras}

Los frutos de guanábana así como las semillas extraídas, fueron caracterizados en función de los marcadores morfológicos internacionalmente establecidos por Bioversity International y CHERLA (2008) adaptados para la especie. Algunos de estos parámetros fueron: longitud, diámetro, peso del fruto, peso de semillas, así como características anatómicas cáscara (exocarpio), pulpa (mesocarpio) y semillas.

\section{Determinación del contenido proximal de la semilla}

La composición química se obtuvo utilizando metodologías oficiales de trabajo establecidas por la AOAC. La determinación de humedad, cenizas, proteínas y extracto etéreo se realizaron según: AOAC 925.1, AOAC 923.03, AOAC 2001.11 y AOAC 920.39, respectivamente. Todos los análisis se realizaron por triplicado con controles negativos (blancos) y controles de calidad internos para la verificación de los resultados. Los datos obtenidos fueron evaluados para determinar los promedios y la desviación estándar de las muestras por triplicado.

\section{Control de calidad de parámetros proximales}

Se incluyeron controles del procedimiento analítico tales como: réplicas de laboratorio, blancos de laboratorio, enriquecidos y agregados. La reproducibilidad de los resultados se evaluó por medio de réplicas de la muestra para demostrar su grado de homogeneidad y representatividad. La precisión de los resultados obtenidos se refleja a través de la desviación estándar relativa (RSD). La exactitud se evaluó a partir de los porcentajes de recuperación obtenidos en los blancos y muestras enriquecidas de semilla de guanábana. Estos se utilizan para demostrar la fiabilidad del método y se consideran aceptables cuando la recuperación del $90 \%$ de los analitos de interés se encuentra entre 90.00 y $105.00 \%$ de la concentración adicionada o enriquecida.

\section{Obtención y mantenimiento del pie de cría de larvas de Spodoptera frugiperda J. E.} Smith

Para garantizar la identidad y salud de los especímenes incluidos en el bioensayo se realizó un pie de cría con masas de huevo del gusano cogollero (10) obtenidas del Laboratorio de Entomología de la Universidad Nacional Autónoma de Nicaragua, León (UNAN-León). Las masas de huevo se mantuvieron en recipientes de policarbonato Magenta ${ }^{\mathrm{TM}}$ hasta el primer estadio larval. Fueron expuestas a fotoperiodos de 12 horas luz y 12 horas oscuridad, con $60 \%$ de 
humedad relativa y $26 \pm 3^{\circ} \mathrm{C}$ de temperatura ambiental. Al alcanzar el segundo estadio larval se individualizaron en tubos de ensayo donde se mantuvieron hasta el tercer estadio larval, cuando se trasladaron a frascos de vidrio. Las larvas se alimentaron cada 24 horas con rectángulos de tejido foliar de maíz previamente desinfectados.

\section{Extracciones por método Soxhlet}

Se pesaron $15 \mathrm{~g}$ de harina de semilla y endospermo de guanábana por duplicado y se colocaron en un dedal de hemicelulosa dentro del equipo Soxhlet donde se adicionaron 100ml del solvente (etanol 96\%) en reflujo durante 16 horas. Los extractos se almacenaron en envases herméticos y oscuros a temperatura ambiente por una semana y posteriormente en refrigeración $\left(4^{\circ} \mathrm{C}\right)$ hasta su utilización.

\section{Preparación de los tratamientos}

Para la aplicación de los extractos (endospermo y semilla completa) se definieron cinco concentraciones o tratamientos: $0.70 \%, 3.00 \%, 7.00 \%, 15.00 \%, 25.00 \%$. El solvente utilizado para su preparación fue agua destilada y desgasificada a $50^{\circ} \mathrm{C}$, manteniendo agitación constante durante su dilución. Los tratamientos se prepararon el mismo día de la aplicación para evitar precipitación o comportamiento bifásico y se almacenaron en contenedores ámbar para evitar su degradación por la luz.

\section{Realización del bioensayo}

Basado en los lineamientos de ensayos entomológicos descritos por Roberson, et al. (2007) y Leatemia \& Isman (2004) adaptados a las condiciones del laboratorio. Las larvas de gusano cogollero se alimentaron con tres rectángulos de tejido foliar de maíz previamente sumergidos en $40 \mathrm{ml}$ de los distintos tratamientos hasta el punto de escurrimiento. Se cambió el alimento cada 24 horas, sin embargo únicamente la primera porción de alimento fue tratada para evitar duplicar la dosis. La mortalidad se determinó en función de la respuesta de las larvas al estímulo con un pincel y se realizaron conteos de mortalidad cada 8 horas. Se verificó también la evidencia de alimentación cada 24 horas, previo al cambio de alimento. El potencial insecticida de cada matriz (endospermo y semilla completa) fue evaluado en los cinco tratamientos antes mencionados $(0.70 \%, 3.00 \%, 7.00 \%, 15.00 \%, 25.00 \%)$. Cada tratamiento fue evaluado en conjuntos de 10 especímenes individualizados en frascos y por triplicado (30 larvas para cada tratamiento), totalizando 150 individuos (300 larvas para ambas matrices).

De igual manera se incluyeron: controles negativos formados por larvas alimentadas con tejido sin tratamiento para corroborar que no existían muertes asociadas a las condiciones de mantenimiento de la especie; control de etanol para desestimar la actividad insecticida del etanol que fue utilizado como solvente en las extracciones; finalmente un control sin alimento 
para verificar la supervivencia de las larvas sin material vegetal. La distribución de las larvas en los controles fue la misma aplicada en los tratamientos (10 larvas individualizadas por triplicado para cada control), totalizando 90 especímenes. Se utilizaron 390 larvas para todo el bioensayo. Cabe señalar que a fin de fomentar la alimentación, los especímenes en estudio no fueron alimentados en 24 horas previas a la realización del bioensayo.

\section{Análisis de datos}

Los datos de la caracterización morfológica de la semilla y fruta se promediaron (datos cuantitativos) y se determinó su frecuencia (datos cualitativos). Los resultados de las réplicas del análisis proximal fueron promediados y comparados con referencias internacionales del Congo (Kimbonguila et al., 2010) y Venezuela (Vit P., Santiago B. y Pérez Pérez E. M., 2014).

Por otro lado, para el análisis del potencial insecticida se determinó la CL50 en base a las mortalidades de cada extracto calculado utilizando la media y mediante el análisis de regresión Probit. La efectividad se estipuló en función del rendimiento obtenido por cada uno de los extractos en base a la mortalidad de las larvas, realizando un análisis de varianza (ANOVA, por sus siglas en inglés) para encontrar la diferencia entre los índices de mortalidad de cada uno. Se consideró como la concentración más efectiva, aquella que en menor concentración elimine al 50\% de los individuos o lo más cerca posible de la mitad. Los supuestos de normalidad y homogeneidad de varianza se realizaron utilizando el programa InfoStat (Balzarini et al., 2008; Di Rienzo et al., 2011), posteriormente se realizaron comparaciones de Fisher para identificar la existencia o ausencia de diferencias significativas entre los grupos.

\section{RESULTADOS Y DISCUSIÓN}

\section{Clasificación morfológica}

Los porcentajes más altos de frecuencia encontrados corresponden a las frutas con forma cordiforme alargada (53.30\%) (Figura 1), exocarpo de protuberancias pequeñas (50.00\%) (Figura 2) y color verde oscuro (36.70\%) (Figura 3 ). 


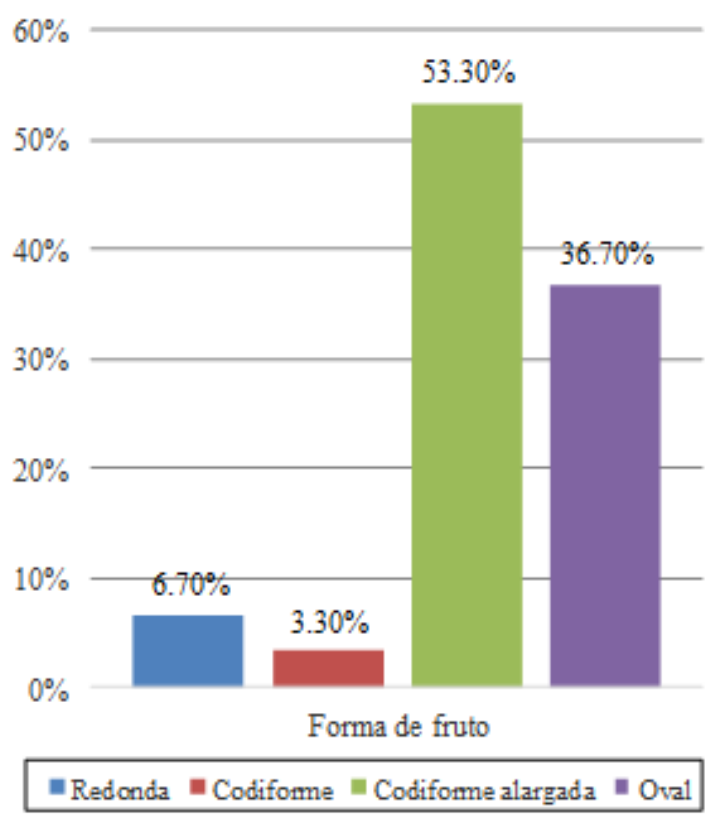

Figura 1. Frecuencias de la forma del fruto.

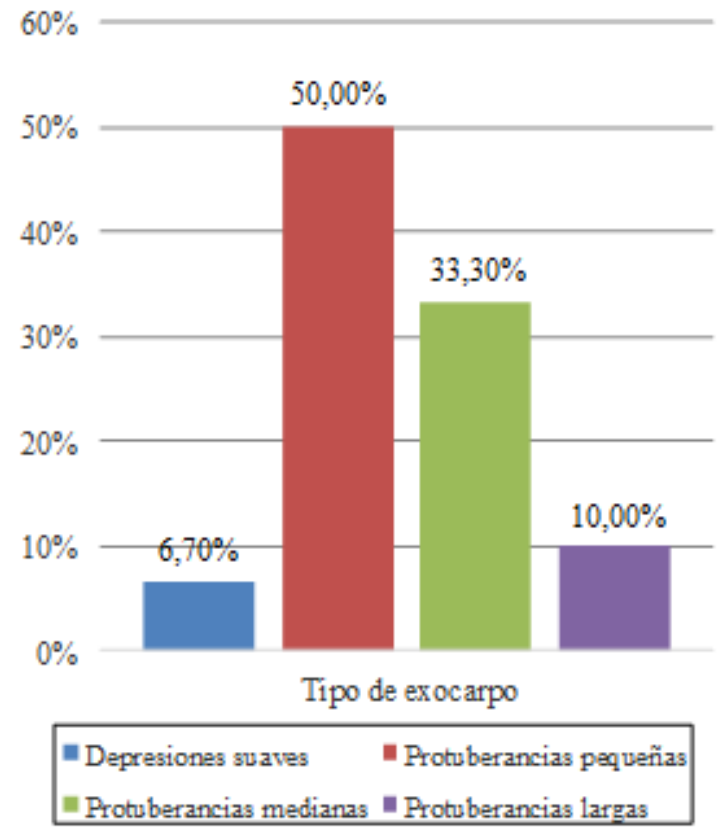

Figura 2. Frecuencias del tipo del exocarpo del fruto. 


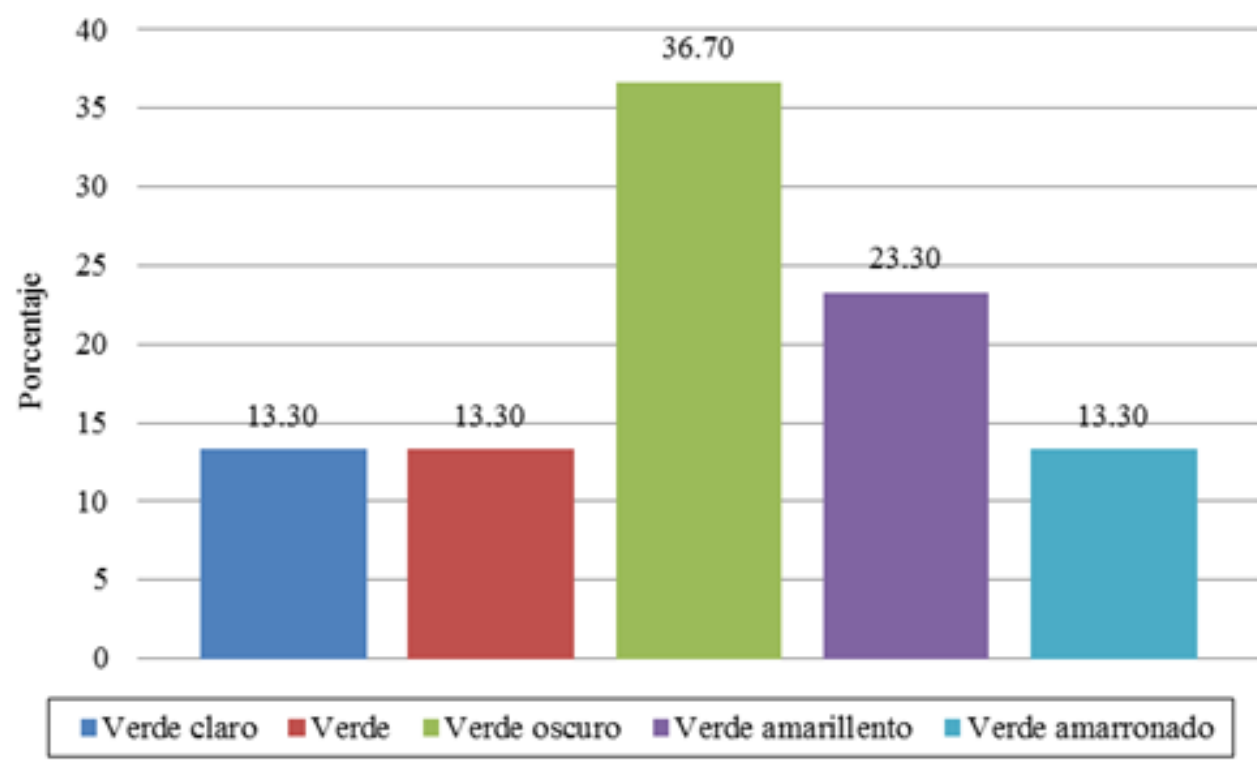

Figura 3. Frecuencias de color de exocarpo.

En relación a las semillas el color predominante fue marrón (80.00\%) (Figura 4) y en el caso de la pulpa el color más preponderante fue blanco (53.30\%) (Figura 5) similar al valor obtenido al sabor dulce (53.30\%) (Figura 6).

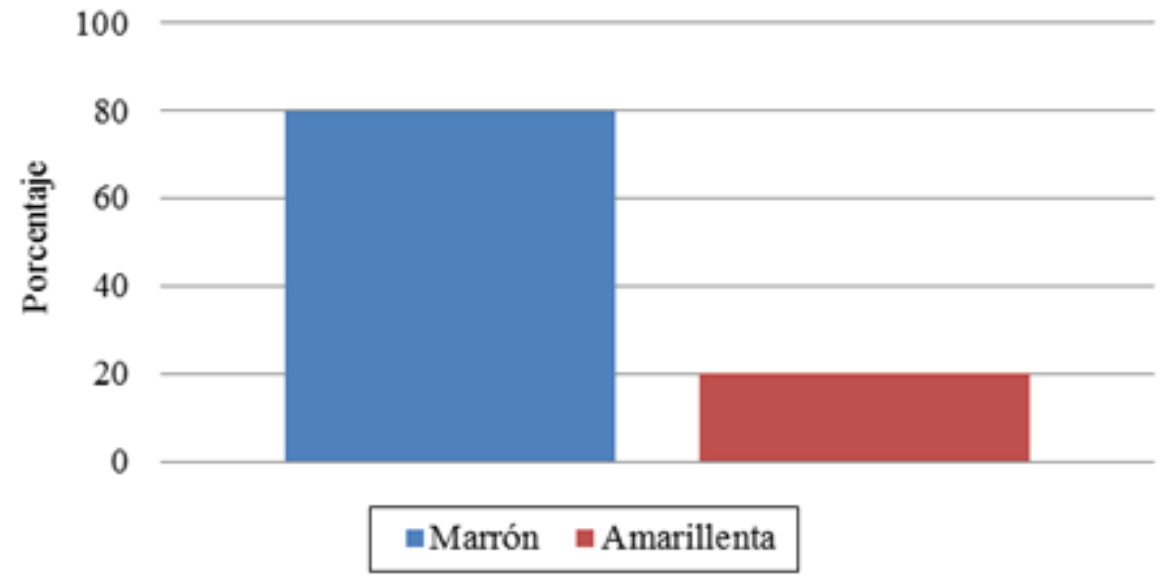

Figura 4. Frecuencia de color de la semilla. 


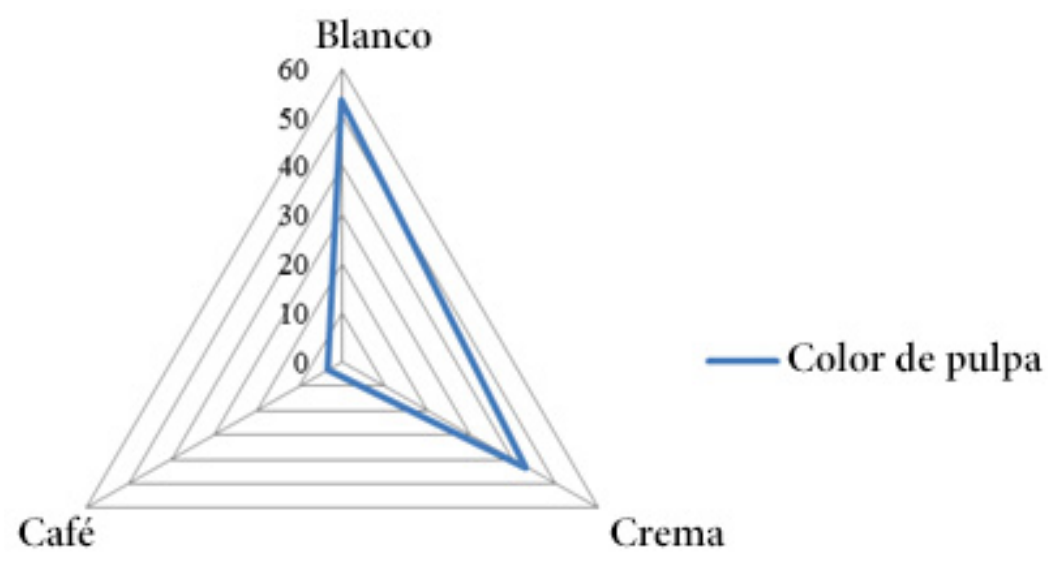

Figura 5. Frecuencias del color de la pulpa.

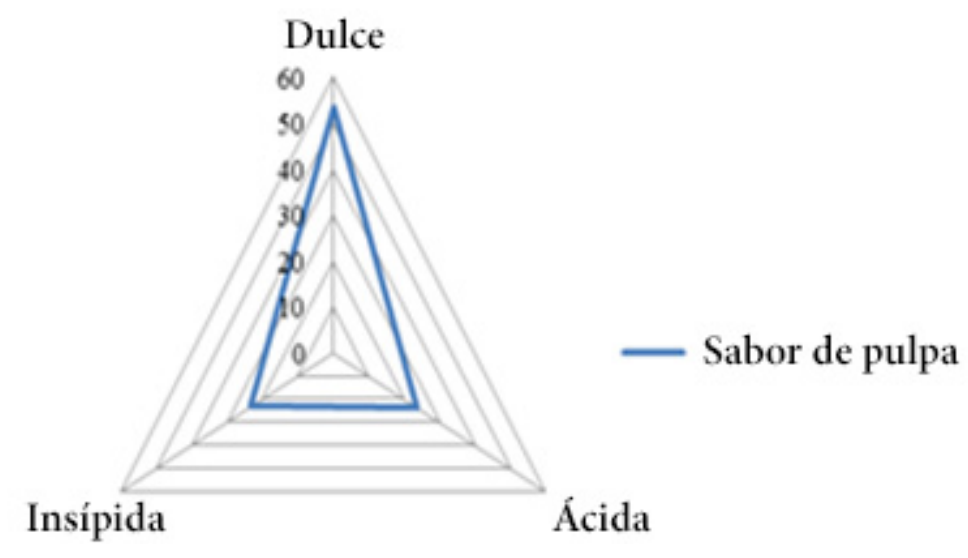

Figura 6. Frecuencias el sabor de la pulpa.

\section{Composición proximal}

El contenido de extracto etéreo $(31.14 \pm 0.56 \%)$ fue el parámetro proximal con mayor predominancia dentro de la materia seca de la semilla de guanábana. En contenidos inferiores $y$ en orden decreciente se encontraron al contenido de proteínas (16.19 $\pm 0.16 \%$ ), nitrógeno $(2.59 \pm 0.16 \%)$ y cenizas $(1.34 \pm 0.03 \%)$ (Figura 7$)$. La humedad y la materia seca determinada fueron: $95.71 \pm 0.02 \%$ y $4.29 \pm 0.02 \%$, respectivamente (Figura 8 ). 


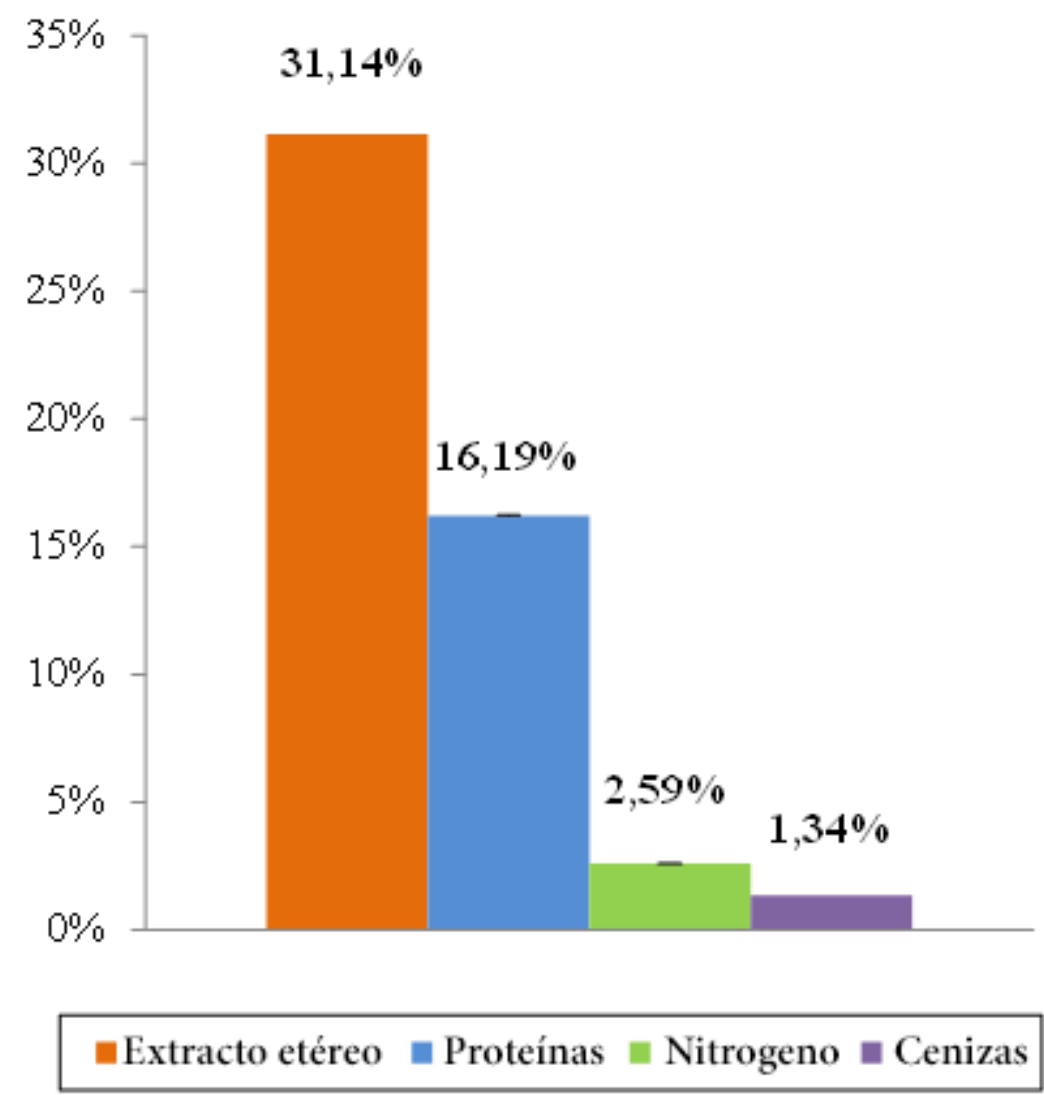

Figura 7. Composición proximal y desviación estándar relativa de la semilla de guanábana.

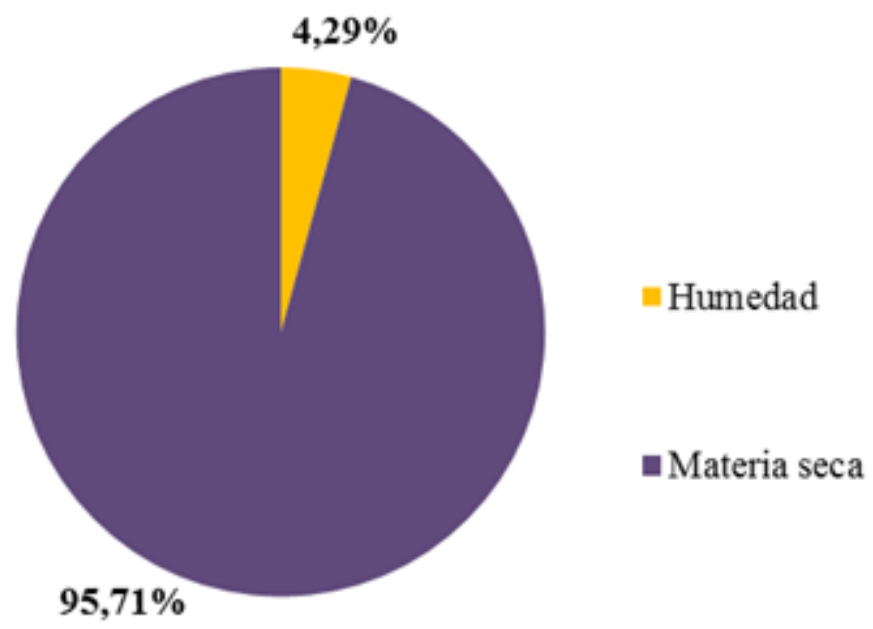

Figura 8: Contenido de humedad y materia seca de la semilla de guanábana.

Al comparar estos resultados con los valores reportados en la literatura se puede observar que la harina de semillas de guanábana de Masaya y Carazo presentan los valores de materia seca más altos y los de humedad más bajos con respecto a harinas de semillas de Mpissa, distrito 
del sur del Congo (Kimbonguila et al., 2010) y Mérida, Venezuela (Vit et al., 2014). La semilla nacional también reporta mayor contenido de grasas totales (extracto etéreo) y proteínas que los obtenidos por Vit et al. (2014), sin embargo, las semillas de Mérida, Venezuela tienen un mayor porcentaje en cuanto a cenizas se refiere (1.44\%). Por otro lado, al comparar los resultados reportados por Kimbonguila et al. (2010) se evidencian valores mayores de cenizas y extracto etéreo $(9.70 \%, y$ y $400 \%$, respectivamente). Este valor considerablemente superior de la fracción lipídica despierta interés en cuanto a su uso en relación a calidad nutricional de los alimentos, aunque reporta un valor menor en proteínas (8.50\%) con relación al obtenido durante los análisis (16.19\%) (Figura 9).

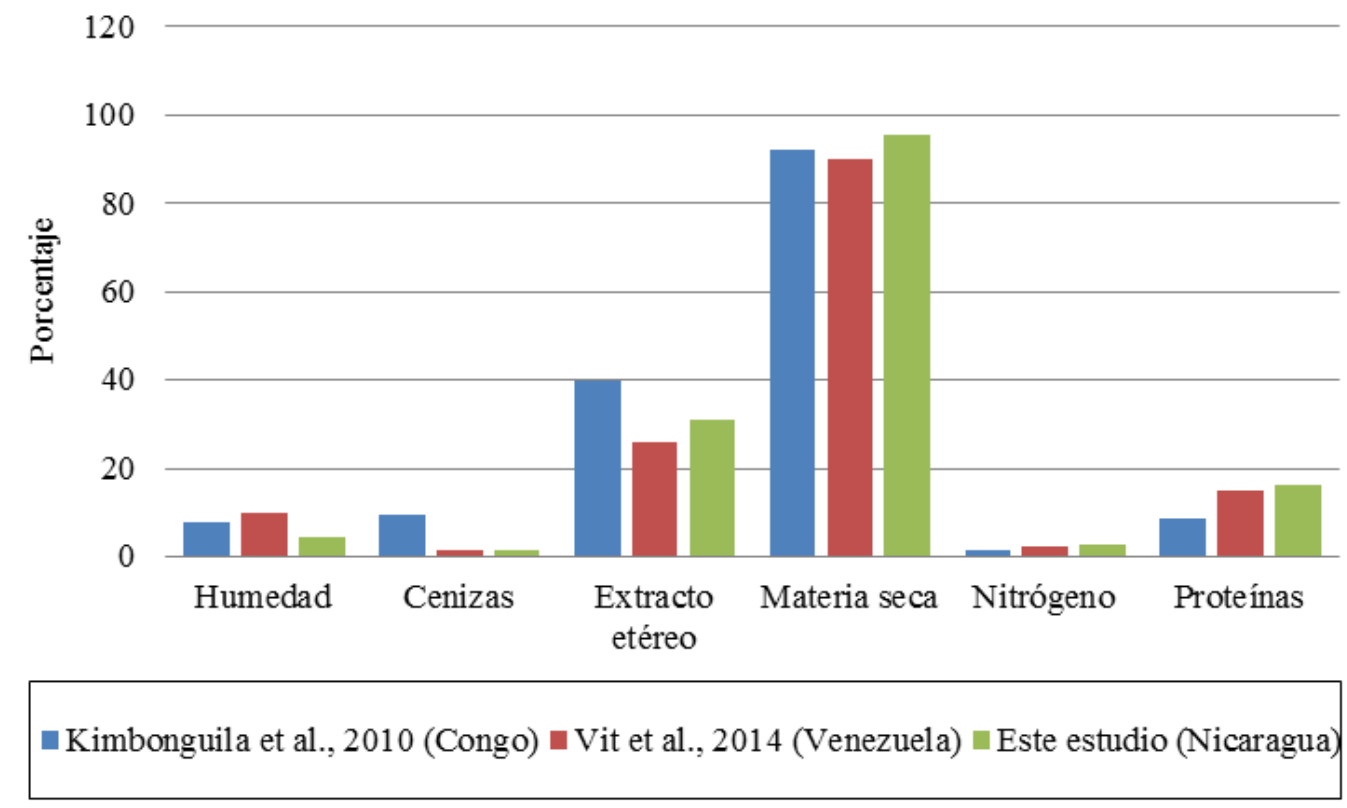

Figura 9. Comparación de la composición de semillas de guanábana en diferentes países.

La variación en los contenidos proximales, a pesar de ser la misma especie, puede deberse a las diferencias en las condiciones climáticas y del suelo de cada país, aportando mayor o menor cantidad de nutrientes a la planta, también influye la variedad de guanábana que se utilizó para los análisis (ácida, sub-ácida o dulce).

\section{Control de calidad de parámetros proximales}

En la tabla 1 se resumen los resultados de los controles de calidad realizados para cada uno de los análisis, los cuales fueron comparados con la guía de interpretación de resultados establecido por la AOAC determinando que todos se encuentran dentro de los rangos permisibles (\% RSD $\leq 1.90$ y \% recobro: 90.00-105.00\%). 
Tabla 1. Controles de calidad de los parámetros proximales en harina de semilla de guanábana.

\begin{tabular}{|l|l|l|l|}
\hline \multirow{2}{*}{ Parámetros } & \multicolumn{1}{|c|}{ Controles de calidad } & \% RSD & \% Recobro \\
\hline \multirow{2}{*}{ Humedad } & Muestra por triplicado & 0.27 & - \\
\cline { 2 - 5 } & Muestra enriquecida (agregado) & 1.62 & 100.55 \\
\hline \multirow{2}{*}{ Cenizas } & Muestra por triplicado & 1.80 & - \\
\hline \multirow{2}{*}{$\begin{array}{l}\text { Extracto } \\
\text { etéreo }\end{array}$} & Muestra por triplicado & 1.80 & - \\
\cline { 2 - 5 } & Muestra enriquecida & 0.03 & 96.96 \\
\cline { 2 - 5 } & Blanco enriquecido & 1.03 & 98.09 \\
\hline \multirow{2}{*}{ Proteínas } & Muestra por triplicado & 0.27 & - \\
\cline { 2 - 5 } & Blanco enriquecido & - & 94.07 \\
\hline
\end{tabular}

\section{Concentración letal media $\left(\mathrm{CL}_{50}\right)$ de los extractos}

La $\mathrm{CL}_{50}$ del extracto etanólico del endospermo de las muestras de semilla de guanábana fue de $2.13 \%$ ( $p=0.0001$ ) (Figura 10). Este resultado muestra que la CL50 está comprendida entre las concentraciones o tratamientos más bajos utilizados para el bioensayo $(0.70 \% \mathrm{y}$ $3.00 \%$ ) que provocaron un efecto letal sobre el $3.33 \%$ y $86.66 \%$, respectivamente. A partir de la concentración de $7.00 \%$ a $25.00 \%$ se registró $100.00 \%$ de mortalidad para este extracto.

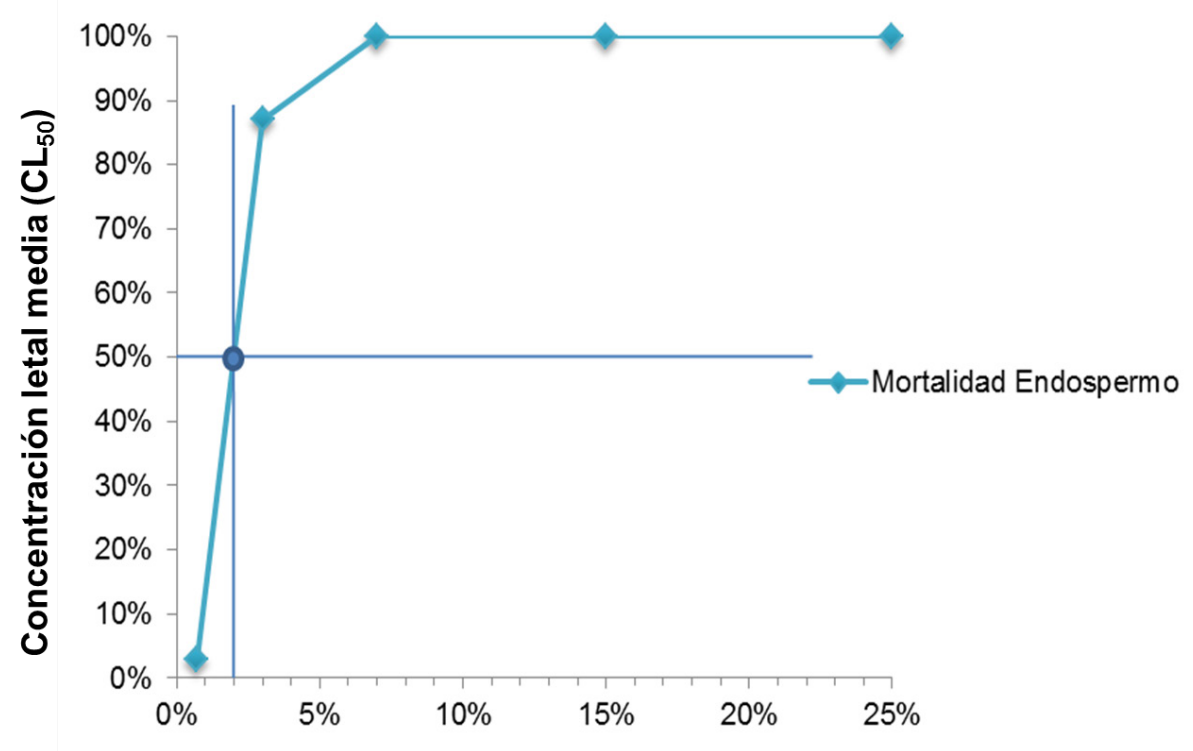

Figura 10. Concentración letal media (CL50) del extracto de endospermo de semillas de Annona muricata L. preparado por el método Soxhlet. 
Para los extractos etanólicos obtenidos a partir de la semilla completa de Annona muricata L. se calculó la CL50 de 1.85 \% ( $p=0.0001$ ) (Figura 11). Este resultado del análisis Probit indica que la $\mathrm{CL}_{50}$ está comprendida entre los tratamientos más bajos utilizados para el bioensayo (0.70\% - $3.00 \%$ ) que provocaron la muerte al 3.33 \% y 96.66 \% de la población, respectivamente. A partir de la concentración de $7.00 \%$ a $25.00 \%$ se registró un $100.00 \%$ de mortalidad para el extracto.

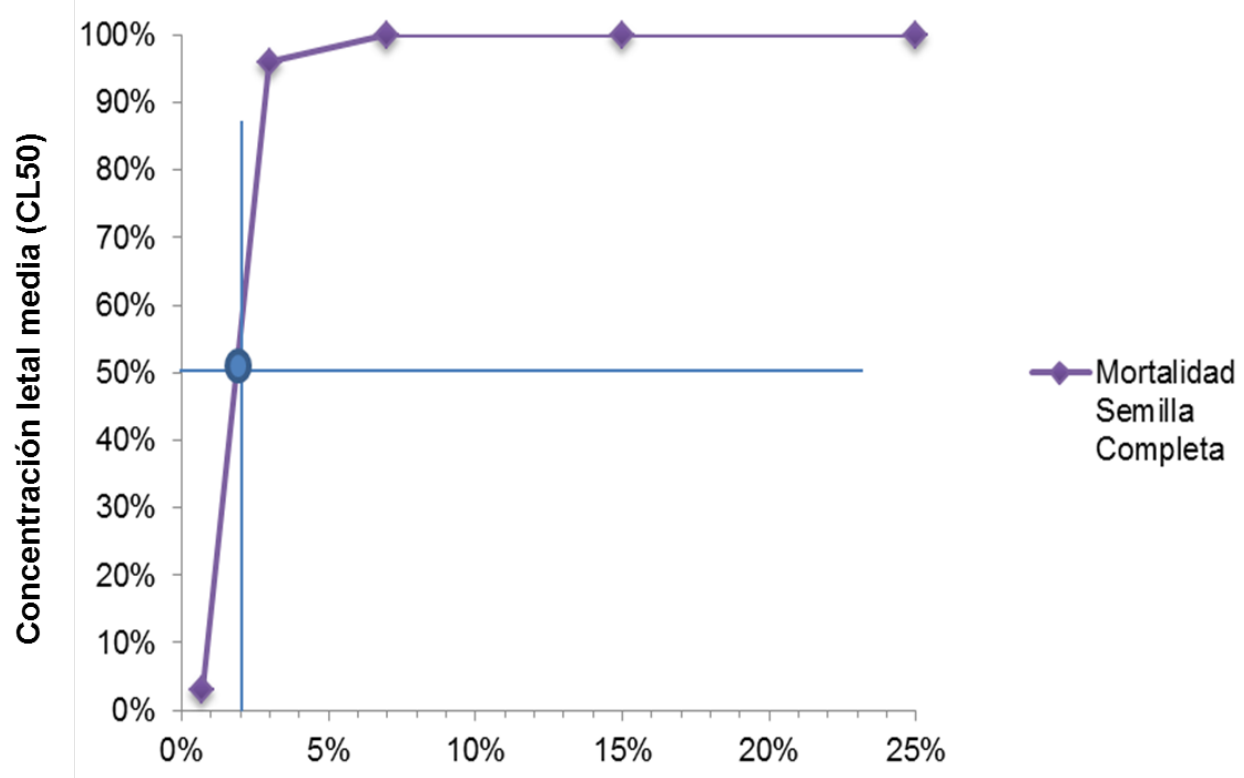

Figura 11. Concentración letal media (CL50) del extracto de semilla completa de Annona muricata L. preparado por el método Soxhlet.

Existe una similitud evidente en los resultados obtenidos por ambos extractos: la CL50 está comprendida entre los tratamientos más bajos del bioensayo y las dosis superiores alcanzan el $100.00 \%$ de letalidad sobre la población de larvas. La diferencia entre los valores de ambos extractos (endospermo y semilla completa) proporciona las pautas para sugerir la semilla completa como el extracto que precisa menor concentración para causar un efecto letal sobre la mitad de la población de insectos en un tiempo de 48 horas después de la aplicación.

Al evaluar los resultados obtenidos con referencias internacionales se identificó la coincidencia con lo establecido por Ángel-Ríos M.D., Pérez-Salgado J. y Morales De Jesús F. (2015) para el efecto del extracto de semilla de A. muricata L. obtenido mediante Soxhlet sobre el gusano cogollero y analizados a una concentración de $2.00 \%$. En este estudio realizado en México se definió que en las condiciones antes descritas los extractos provocaban el $87.97 \%$ de mortalidad sobre las larvas, similar a la mortalidad obtenida en el tratamiento de $3.00 \%$ en la presente investigación (96.66\%). 
La toxicidad aguda se analizó en base a los datos colectados durante el bioensayo (48 horas), sin embargo, la concentración mediana letal no fue una constante a través del tiempo, razón por la cual se establecieron varios tiempos de conteo (cada 8 horas) y se calculó la CL50 de acuerdo a los principios ecotoxicológicos de Walker, C. H., Sibly, R. M., Hopkin, S. P., \& Peakall, D. B. (2012). La curva de la Figura 12 muestra como la toxicidad aguda disminuye a medida que el tiempo de exposición aumenta hasta alcanzar la concentración umbral cerca de las 48 horas donde la curva empieza a paralelizarse con la asíntota $\mathrm{X}$, no obstante, entre los dos últimos tiempos de conteo (40-48 horas) se distingue una variación de las CL50 de $2.20 \%$ a $2.13 \%$ respectivamente, indicando que la concentración umbral no es definitiva y que la mortalidad puede variar con el incremento del tiempo de observación (más de 48 horas), hasta que la toxicidad aguda deje de variar a través del tiempo convirtiéndose en una recta.

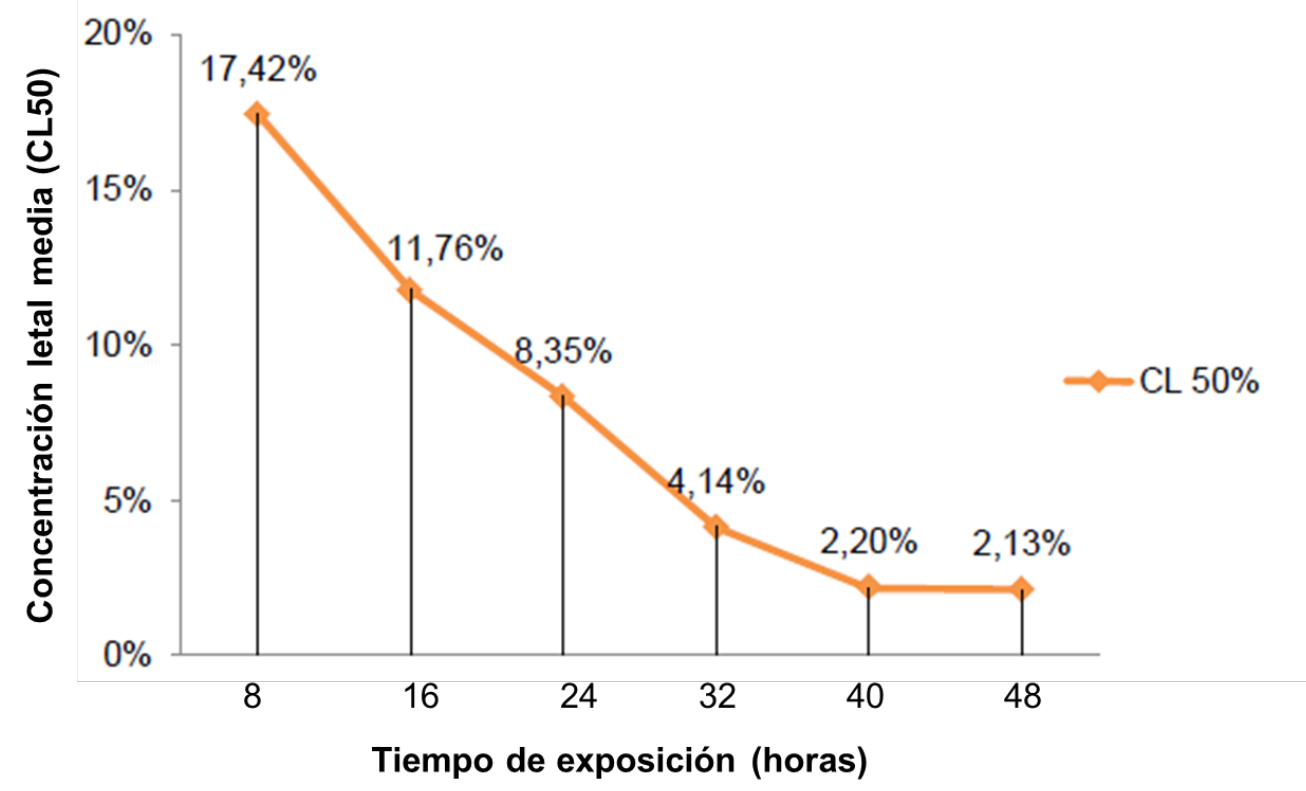

Figura 12. Concentración letal media (CL50) en los tiempo de exposición del extracto de endospermo de semillas de Annona muricata L. sobre las larvas de Spodoptera frugiperda.

En la curva de la concentración mediana letal a través de los tiempos de exposición durante las 48 horas para el extracto de semilla completa (Figura 13) se apreció como la curva decrece hasta las 40 horas y, opuesto a lo ocurrido con el extracto de endospermo, se adquiere un comportamiento totalmente lineal con respecto al eje $\mathrm{X}$ alcanzando su concentración umbral definitiva, es decir que no se experimentarán alteraciones en la mortalidad aunque se incremente el tiempo de exposición, cumpliendo perfectamente con los principios ecotoxicológicos de Walker et al. (2012). Cuando el umbral se alcanza todo el sistema experimental se estabiliza, por tanto la concentración de toxinas en el organismo de las larvas no podía aumentar o disminuir sin importar si ingerían alimento o no, durante y después de las 48 horas, así los sujetos que 
lograron sobrevivir al bioensayo, sobrevivieron a través del tiempo sobrellevando las toxinas en sus metabolismos.

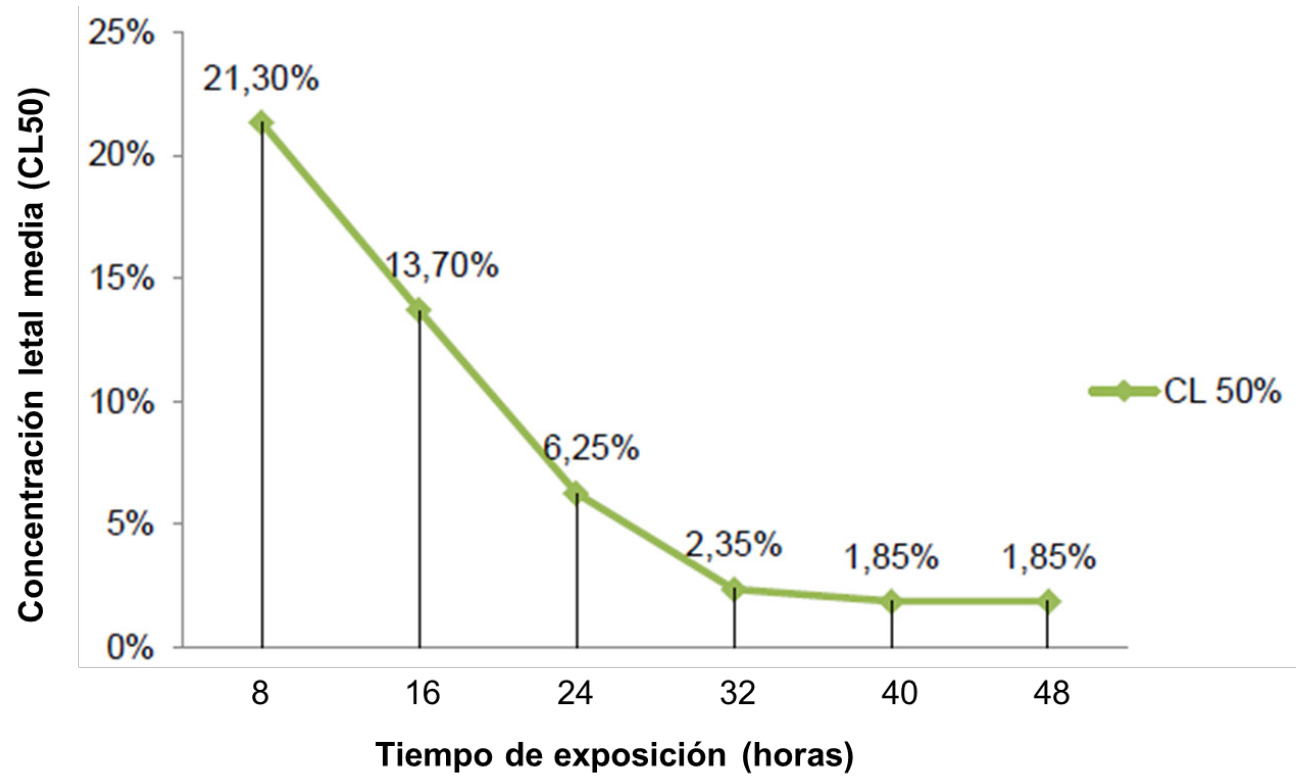

Figura 13. Concentración letal media (CL50) en los tiempos de exposición del extracto de semilla completa de Annona muricata L. sobre las larvas de Spodoptera frugiperda.

El intervalo de tiempo que registró mayor mortalidad fue 8 horas después de la aplicación de los extractos con un total de 110 larvas (73.33\%) para el extracto de endospermo y 113 larvas (75.33\%) para semilla completa.

La determinación de la $\mathrm{CL}_{50}$ indica que tanto el extracto de endospermo como semilla completa poseen la toxicidad necesaria para causar la muerte del 50\% de la población de larvas a dosis bajas e incluso a la totalidad de la población en concentraciones superiores al 3.00\%, sin embargo a fin de verificar su actividad in situ se debe realizar una validación en campo con una concentración estandarizada, principalmente del extracto de semilla completa que resultó ser el más eficiente al requerir menor concentración para alcanzar la $C_{50}$.

\section{Efecto anti-alimentario}

Durante el bioensayo se registró la ingesta de alimento por parte de las larvas y se observó que existe un comportamiento inverso entre la concentración del extracto y la evidencia de ingesta del tejido foliar de maíz utilizado como alimento: al incrementarse la concentración o tratamiento se registraba mayor mortalidad con menor evidencia de alimentación. Esta observación permitió inferir que los extractos tienen un efecto repelente o anti-alimentario sobre las larvas y combinado con su toxicidad por contacto ocasionó la muerte de las larvas, coincidiendo con lo reportado por Leatemia e Isman (2004). De esta manera se puede observar 
que los extractos actúan por ingesta, por contacto o repelencia, elevando el potencial de la $A$. muricata L. como una alternativa para el control del gusano cogollero.

\section{ANOVA de las concentraciones aplicadas a las larvas}

El análisis de varianza y la comparación de Fisher mostraron que las concentraciones de los dos extractos (endospermo y semilla completa) comprendidas entre 3.00 y $25.00 \%$ no presentaron diferencias significativas entre las medias de las mortalidades de las larvas de $S$. frugiperda, pero sí existe diferencia entre éstas y la concentración de $0.70 \%$ que resultó significativamente inferior ( $p=0.0001, \mathrm{~F}=35.85$ ) (Figura 14).

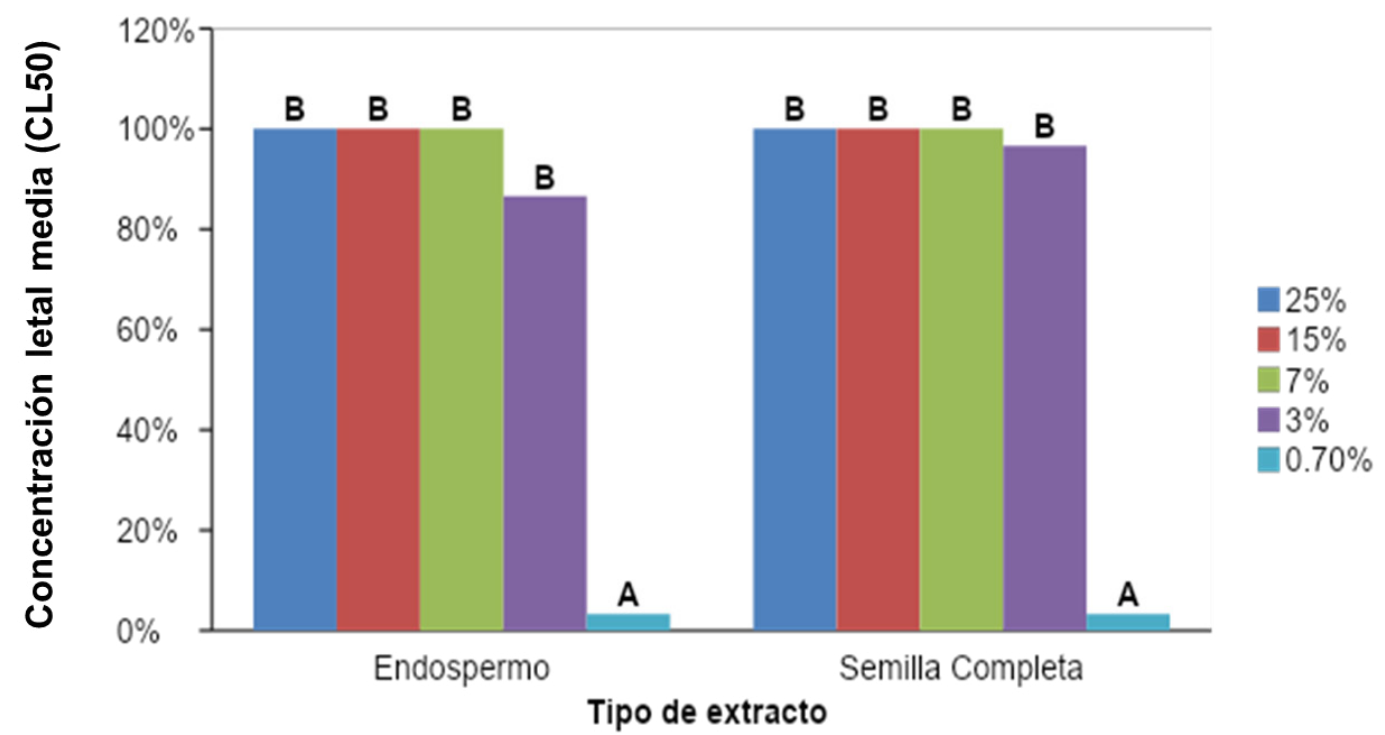

Figura 14. Comparación de las medias de las mortalidades reportadas para los distintos tratamientos preparados a partir de extractos de endospermo y semilla completa de A. muricata L. sobre las larvas de S. frugiperda. Los tratamientos identificados con la misma letra no difieren estadísticamente.

La similitud entre las mortalidades registradas para ambos extractos puede deberse a que las acetogeninas, causantes del efecto insecticida, se encuentran distribuidas de manera similar en ambas matrices (semilla completa y endospermo) debido a que es en estas porciones donde se encuentra concentrada la porción lipídica de la semilla de guanábana. Cabe señalar que durante la preparación de los tratamientos, ambas matrices presentaron el mismo comportamiento bifásico en concentraciones altas (15.00 y 25.00\%).

\section{CONCLUSIONES}

La caracterización morfológica de las muestras analizadas reflejó como características predominantes la forma alargada (53.30\%), exocarpo con protuberancias pequeñas (50.00\%) y color verde oscuro (36.70\%). De igual manera, las semillas contenidas en el fruto son 
preponderantemente marrones $(80.00 \%)$ y la pulpa posee color blanco con un sabor dulce (53.33\%).

El análisis proximal de la semilla reflejó un $1.34 \%$ de cenizas superado por valores reportados en muestras de Mpissa, Congo, 4.29 \% de humedad y 95.71 \% de materia seca, 31.14 \% de extracto etéreo, 2.59 \% de nitrógeno y $16.19 \%$ de proteína, siendo estos últimos similares a las referencias internacionales publicadas de Mpissa, Congo y Mérida, Venezuela.

La CL50 para el extracto de endospermo fue $2.13 \%$ y para el extracto de semilla completa fue $1.85 \%$, identificando a esta última como la alternativa más eficiente para el control del gusano cogollero por requerir menor concentración para causar la mortalidad del 50\% de la población. De igual manera, durante el bioensayo se evidenció efecto anti-alimentario de los extractos con una tendencia inversa al incremento de las concentraciones.

\section{AGRADECIMIENTOS}

Este estudio se realizó dentro del Marco del Convenio Interinstitucional establecido con el Instituto Nicaragüense de Tecnología Agropecuaria (INTA) con apoyo del financiamiento recibido por el Fondo para Proyectos de Investigación (FPI) de UNAN-Managua. Especial agradecimiento a la Dra. Katia Montenegro Rayo por la asesoría brindada en la revisión de los resultados obtenidos.

\section{REFERENCIAS BIBLIOGRÁFICAS}

Ángel-Ríos M.D., Pérez-Salgado J. y Morales

De Jesús F. (2015). Toxicidad de extractos vegetales y hongos entomopatogenos en el gusano cogollero Spodoptera frugiperda J.E.Smith (Lepidoptera: noctuidae), del maíz en el estado de Guerrero. Entomología Mexicana Vol. 2: 260-265 (2015).

Arroyo A, J., Prashad G, M., Vásquez B., Y., Li P, E., \& Tomás C, G. (2005). Actividad citotóxica in vitro de la mezcla de Annona muricata y Krameria lappacea sobre células cancerosas de glándula mamaria, pulmón y sistema nervioso central. Revista Peruana de Medicina Experimental y Salud Publica, 22(4), 247-253.
Balzarini, M. G., Gonzalez, L., Tablada, M., Casanoves, F., Di Rienzo, J. A., \& Robledo, C. W. (2008). Manual del Usuario. Córdobas, Argentina: Editorial Brujas.

Bobadilla , M., Zavala, F., Sisniegas, M., Zavaleta, G., Mostacero, J., \& Taramona, L. (2005). Evaluación larvicida de suspensiones acuosas de Annona muricata Linnaeus «guanábana» sobre Aedes aegypti Linnaeus (Diptera, Culicidae). Revista peruana de Biología, 12(1), 145-152.

Di Rienzo, J. A., Casanoves, F., Balzarini, M. G., Gonzalez, L., Tablada, M., \& Tablada, Y. C. (2011). InfoStat versión 2011. 8, 195199. Argentina. Recuperado de http:// www.infostat.com.ar 
Espinoza, S. A., Urbina, A. R., Obando, S. R., \& Vanegas, J. R. (1999). Cultivo del maíz. Managua, Nicaragua: Instituto Nicaragüense de Tecnología Agropecuaria. Recuperado de http:// agris.fao.org/agris-search/search. do?recordID =NI2006002701

FAO. 2019. Datos sobre la tierra en http:// www.fao.org/faostat/en/\#data/RL, accesado el 24 de marzo del 2019.

INTA. (2010). Cultivo del maiz. Guía Tecnológica 3, Instituto Nicaragüense de Tecnología Agropecuaria, Managua.

Jaramillo D., Á., Jaramillo G., O., Bustillo P., A., \& Gómez L., H. (1989). Efecto del Gusano Cogollero Spodopfera frugiderpa (J. E. Smith) sobre el Rendimiento del Maíz. Revista Facultad Nacional de Agronomía Medellín, 42(1), 25-33.

Kimbonguila A., Nzikou J. M., Matos L., Loumouamou B., Ndangui C. B., Pambou-Tobi N., et al.. (2010). Proximate Composition and Physicochemical Properties on the Seeds and Oil of Annona muricata grown in Congo-Brazzaville. Research Journal of Enviromental and Earth Sciences, 2(1), 13-18.

Leatemia, J. A., \& Isman, M. B. (2004). muricata). Plant Foods for Human Nutrition, 57(2), 165-171.

Pardo Abdala, Lissett María, Pérez Rodríguez, Sonia, \& Gámez Bacallao, Arianna. (2017). Reportes al Centro Nacional de Toxicología de mujeres en edad fértil expuestas a plaguicidas. Revista Cubana de Medicina Militar, Volumen 46, número 1, páginas 10-18.

Peckham, G. (1979). Foundations of Food Preparation. New York, Estados Unidos : MCMILLAN, 1979. Ref 641.5 P368 1979.

Roberson, J. L., Savin, N. E., Russell, R. M., \& Preisler, H. K. (2007).Bioassays with Arthropods (Segunda ed.). (T. a. Group, Ed.) Estados Unidos: CRC Press.

Santos Pimenta, L.P., Pinto G.B., TakahashiJ.A., Silva L.G.F. e, Boaventura M.A.D. (2003). Biological screening of Annonaceous Brazilian Medicinal Plants using Artemia salina (Brine Shrimp Test), Phytomedicine, Volume 10, Issues 2-3, 2003, Pages 209-212, ISSN 0944-7113, https://doi. org/10.1078/094471103321659960.

Vit P., Santiago B. y Pérez Pérez E. M. (2014). Composición química y actividad antioxidante de pulpa, hoja y semilla de guanábana (Annona muricata L.). Interciencia, 39(5), 350-353.

Walker, C. H., Sibly, R. M., Hopkin, S. P., \& Peakall, D. B. (2012). Principles of ecotoxicology (4th Edition). Florida, Estados Unidos: Taylor \& Francis Group. ISBN 9781439862667. 